\title{
High-Frequency Scattering From a Coated Sphere ${ }_{j}^{1}$
}

\author{
V. H. Weston and R. Hemenger \\ Contribution from Conductron Corporation, Ann Arbor, Mich.
}

(Received April 23, 1962; revised May 1, 1962)

\begin{abstract}
The scattered field produced by a plane wave incident on a perfectly conducting sphere coated with a thin layer of material with large complex index of refraction is considered. It is shown that, for certain relations involving the thickness of the layer, and its permittivity and permeability, the scattering problem is equivalent to the problem wherein the total electric and magnetic field components must satisfy a general impedance boundary condition on the outer surface. With this simplification the backscattered field is obtained in terms of the geometric optics portion and the diffracted field portion for small wavelengths.
\end{abstract}

\section{Introduction}

Previous work on scattering by spheres comprised of concentric layers of various materials has been carried out by Aden and Kerker [1951], and Sharfman [1954], who obtained the exact Mie series for the various cases they considered. However, they did not perform an asymptotic evaluation of the fields for the small wavelength case.

In this paper the investigation for small wavelengths is considered for the ease where the particular diffracting body is a perfectly conducting sphere coated with a thin layer of large complex refractive index. First the exact Mie series is given for the scattered field. Using asymptotic methods it is shown that the effect of the coating can be replaced by an equivalent impedance boundary on the outer surface. In so doing, certain restrictions are placed on the thickness of the coating and the argument of the index of refraction.

A similar problem, namely that of a radial electric dipole, outside a concentrically stratified spherical conductor was treated by Wait [1956]. An approximation obtaining the impedance boundary condition was also discussed by Wait.

\section{Mie Series}

Consider a perfectly conducting sphere with radius $b$ covered by a homogeneous layer with uniform thickness $\delta$ of a material characterized by its electric permittivity $\epsilon$ and relative magnetic permeability $\mu^{\prime}$-which may be complex - on which is incident the plane electromagnetic wave

where

$$
\begin{aligned}
& E_{x}=E_{0} e^{-i \omega t+i k z}, \\
& H_{y}=\eta_{0} E_{0} e^{-i \omega t+i k z}
\end{aligned}
$$

$$
\begin{aligned}
k & =\frac{2 \pi}{\lambda}=\frac{\omega}{c}=\omega\left(\epsilon_{0} \mu_{0}\right)^{1 / 2}, \\
\eta_{0} & =\left(\epsilon_{0} / \mu_{0}\right)^{1 / 2}
\end{aligned}
$$

and $\epsilon_{0}$ and $\mu_{0}$ are the permittivity and permeability of the surrounding medium, taken to be free space. The wave number of the coating is given by $k_{1}$ and is related to the index of refraction by the relation

$$
k_{1}=N k \text {. }
$$

1 The work described in this paper was carried out for the Air Force Ballistic Missile Division under subcontract to the Conductron Corporation as a part of the program being carried out under Chrysler Corporation Prime Contract A FO4(694)-25. 
The general Mie series solution for a sphere with a concentric shell is given by Aden and Kerker [1951] and the case where the inner sphere is a perfect conductor by Sharfman [1954]. Using these results the far scattered field is thus found to be, in spherical components form,

$$
\begin{gathered}
E_{r}=H_{r}=0 \\
E_{\theta}=\frac{1}{\eta_{0}} H_{\varphi}=-\frac{i}{k r} E_{0} e^{-i \omega t+i k r} \sum_{n=1}^{\infty} \frac{2 n+1}{n(n+1)}\left(a_{n} \frac{P_{n}^{1}}{\sin \theta}+b_{n} \frac{\partial P_{n}^{1}}{\partial \theta}\right) \cos \varphi \\
E_{\varphi}=-\frac{1}{\eta_{0}} H_{\theta}=\frac{i}{k r} E_{0} e^{-i \omega t+i k r} \sum_{n=1}^{\infty} \frac{2 n+1}{n(n+1)}\left(a_{n} \frac{\partial P_{n}^{1}}{\partial \theta}+b_{n} \frac{P_{n}^{1}}{\sin \theta}\right) \sin \varphi
\end{gathered}
$$

where

$$
\begin{aligned}
& a_{n}=-\frac{\psi_{n}(k a) A_{n}-\psi_{n}^{\prime}(k a)}{\zeta_{n}^{(1)}(k a) A_{n}-\zeta_{n}^{\prime(1)}(k a)} \\
& b_{n}=-\frac{\psi_{n}(k a) B_{n}-\psi_{n}^{\prime}(k a)}{\zeta_{n}^{(1)}(k a) B_{n}-\zeta_{n}^{\prime(1)}(k a)}
\end{aligned}
$$

and $\psi(k a)$ and $\zeta(k a)$ are related to the Bessel functions by

$$
\psi_{n}(x)=\sqrt{\frac{x \pi}{2}} J_{\nu}(x), \quad \zeta_{n}^{(1),(2)}(x)=\sqrt{\frac{x \pi}{2}} H_{\nu}^{(1),(2)}(x)
$$

with $\nu=n+1 / 2$.

The radius of the outer sphere is given by $a=b+\delta$. The functions $A_{n}$ and $B_{n}$ are given by

with

$$
\begin{gathered}
A_{n}=\frac{N}{\mu^{\prime}} \frac{\partial\left(\ln C_{n}\right)}{\partial\left(k_{1} a\right)} \\
B_{n}=\frac{\mu^{\prime}}{N} \frac{\partial}{\partial\left(k_{1} a\right)}\left[\ln \frac{\partial C_{n}}{\partial\left(k_{1} b\right)}\right]
\end{gathered}
$$

$$
C_{n}=\zeta_{n}^{(2)}\left(k_{1} a\right) \zeta_{n}^{(1)}\left(k_{1} b\right)-\zeta_{n}^{(2)}\left(k_{1} b\right) \zeta_{n}^{(1)}\left(k_{1} a\right)
$$

\section{Impedance Boundary Condition Approximation}

It will be shown that for large $k a, N, k b$, and certain other conditions, the exact Mie series for the scattered field can be approximated by a Mie series for which the total field on the surface of the sphere satisfies the generalized impedance boundary condition, Leontovich [appendix to the Logan and Blacksmith collection of Fock papers, 1957]

$$
\mathbf{E}-(\mathbf{n} \cdot \mathbf{E}) \mathbf{n}=\eta \eta_{0}^{-1} \mathbf{n} \times \mathbf{H}
$$

where the relative impedance $\eta$ is a function of $\mu^{\prime}, \epsilon$, and $\delta$.

The procedure is centered around the asymptotic approximations to the coefficients $A_{n}$ and $B_{n}$. First the coefficient $C_{n}$ is considered. It is written in the following two forms

$$
\begin{aligned}
C_{n} & =\frac{\pi}{2} \sqrt{k_{1} a k_{1} b}\left\{2 J_{\nu}\left(k_{1} a\right) H_{\nu}^{(1)}\left(k_{1} b\right)-2 J_{\nu}\left(k_{1} b\right) H_{\nu}^{(1)}\left(k_{1} a\right)\right\} \\
& =\frac{\pi}{2} \sqrt{k_{1} a k_{1} b}\left\{H_{\nu}^{(2)}\left(k_{1} a\right) H_{\nu}^{(1)}\left(k_{1} b\right)-H_{\nu}^{(2)}\left(k_{1} b\right) H_{\nu}^{(1)}\left(k_{1} a\right)\right\}
\end{aligned}
$$

where $\nu=n+1 / 2$. Before considering the asymptotic evaluation of the Hankel functions for large order, the following restriction will be placed upon the argument of $N$,

$$
\emptyset \leq \arg N<\frac{\pi}{2}-\emptyset^{\prime}
$$


where the positive angles $\emptyset$ and $\emptyset^{\prime}$ satisfy the inequalities

$$
\begin{aligned}
& \emptyset>-|N k b|^{-2 / 3} \\
& \emptyset^{\prime}>>|N k b|^{-1} .
\end{aligned}
$$

The lower bound on the argument of $N$ insures that the following inequalities hold,

$$
\begin{aligned}
& \left|k_{1} a-\nu\right|>\nu^{1 / 3} \\
& \left|k_{1} b-\nu\right|>\nu^{1 / 3}
\end{aligned}
$$

for the complete range of $n$ of interest. Thus the Debye asymptotic expansions for the Bessel and Hankel functions may be used. Hence from Watson [ch. 8, 1958], the coefficient $C_{n}$ has the following asymptotic form for large $N k a$ and $N k b$,

where

$$
C_{n} \sim \frac{2}{\sqrt{\left(-i \sinh \gamma_{1}\right)\left(-i \sinh \gamma_{2}\right)}} \sinh \left[\psi_{2}-\psi_{1}\right]
$$

with

$$
\left.\begin{array}{l}
\nu=N k a \cosh \gamma_{1} \\
\nu=N k b \cosh \gamma_{2}
\end{array}\right\}
$$

$$
\gamma_{1}=\alpha_{1}+i \beta_{1} \text {, and } \gamma_{2}=\alpha_{2}+i \beta_{2} \text {. }
$$

Both $\alpha_{1}$ and $\alpha_{2}$ are negative and both $\beta_{1}$ and $\beta_{2}$ lie between 0 and $\pi / 2$. The functions $\psi$ are given by

$$
\left.\begin{array}{l}
\psi_{1}=\nu\left(\tanh \gamma_{1}-\gamma_{1}\right) \\
\psi_{2}=\nu\left(\tanh \gamma_{2}-\gamma_{2}\right)
\end{array}\right\}
$$

Expression (10) is the leading term of an asymptotic expansion, provided that condition (8) holds. If the argument of $N$ is the order of, or less than $\emptyset$, then the Airy integral approximation would have to be used for the Bessel functions when $\nu /(N k a)$ or $\nu /(N k b) \sim 1$. If the argument of $N$ is the order of, or greater than $\pi / 2-\emptyset^{\prime}$, then expression (10) would have to be modified by the addition of extra terms when $\nu /(N k a)$ or $\nu /(N k b)$ is in the vicinity of $-1.5 i$.

Without going into their derivation, certain properties of the functions associated with the right-hand side of eq (10) will be given. First, it can be shown that the real part of $\psi_{2}-\psi_{1}$ is a positive function and monotonically increases with increasing real $\nu$. The imaginary part of $\psi_{2}-\psi_{1}$ is negative and monotonically increases with increasing real $\nu$. The real part of $\sinh \gamma_{1}$ and $\sinh \gamma_{2}$ are both negative, whereas their imaginary parts are both positive.

From (3) and (10) the asymptotic behavior of $A_{n}$ for large $N, k a, k b$ is given by

$$
A_{n} \sim-\frac{N}{\mu^{\prime}} \operatorname{coth}\left(\psi_{2}-\psi_{1}\right) \sinh \gamma_{1}
$$

and similarly for $B_{n}$

$$
B_{n} \sim-\frac{\mu^{\prime}}{N} \tanh \left(\psi_{2}-\psi_{1}\right) \sinh \gamma_{1}
$$

In particular for $n<<|N k b|$, these become

$$
\begin{aligned}
& A_{n} \sim-\frac{i N}{\mu^{\prime}} \operatorname{coth}\left(\psi_{2}-\psi_{1}\right)\left\{1+0\left(\frac{n}{k_{1} b}\right)^{2}+0\left(\frac{\delta}{k_{1} a^{2}}\right)\right\} \\
& B_{n} \sim-\frac{i \mu^{\prime}}{N} \tanh \left(\psi_{2}-\psi_{1}\right)\left\{1+0\left(\frac{n}{k_{1} b}\right)^{2}+0\left(\frac{\delta}{k_{1} a^{2}}\right)\right\}
\end{aligned}
$$

where the term which is the order of $\delta /\left(k_{1} a^{2}\right)$ arises from the consideration of higher order terms in the asymptotic expansion for $A_{n}$ and $B_{n}$. Finally one obtains 


$$
\psi_{2}-\psi_{1}=-i k_{1} \delta+i k_{1} \delta \frac{a}{2 b}\left(\frac{n+1 / 2}{k_{1} a}\right)^{2}+0\left[\frac{\delta}{b}\left(\frac{n}{k_{1} a}\right)^{2}\right]
$$

Hence for the range of $n, 0<n<k a M$ where $M$ is a number unspecified as yet but lying in the range $1<M<<|N|$, the following approximation holds,

$$
\psi_{2}-\psi_{1} \sim-i k_{1} \delta
$$

provided that $\delta$ is sufficiently small such that

$$
M^{2}\left|\frac{k_{1} \delta}{2 N^{2}}\right|<<1
$$

Thus for the range $0<n<M k a$, the constants $A_{n}$ and $B_{n}$ are effectively independent of $n$. Hence set

$$
B_{n}=-i \eta
$$

Using the relation $A_{n} \sim-\left(B_{n}\right)^{-1}$ the set of coefficients $a_{n}$ and $b_{n}$ of the Mie series (eq (21)) can be approximated by the following,

$$
\begin{aligned}
& a_{n}=-\frac{\psi_{n}(k a)-i \eta \psi_{n}^{\prime}(k a)}{\zeta_{n}^{(1)}(k a)-i \eta \zeta_{n}^{(1)}(k a)} \\
& b_{n}=-\frac{i \eta \psi_{n}(k a)+\psi_{n}^{\prime}(k a)}{i \eta \zeta_{n}^{(1)}(k a)+\zeta_{n}^{(1)}(k a)} .
\end{aligned}
$$

But these are the corresponding coefficients that one would obtain for a boundary condition of the type given by eq (6) [see the appendix of Hiatt et al., 1960].

The remaining problem is to consider the behavior of the coefficients for $n>M k a$. It will be assumed that $M$ is sufficiently larger than unity so that the Debye asymptotic expansions may be used for $\psi_{n}(k a)$ and $\zeta_{n}^{(1)}(k a)$ for $n>M k a$. Explicity $M$ must be such that $M-1>>$ $(k a)^{-2 / 3}$. Thus one obtains

$$
\begin{gathered}
\psi_{n}(k a) \sim \frac{\exp \{\nu(\tanh \gamma-\gamma)\}}{\sqrt{\sinh \gamma}} \\
\zeta_{n}^{(1)}(k a) \sim \frac{\exp \{-\nu(\tanh \gamma-\gamma)\}}{\sqrt{\sinh \gamma}}
\end{gathered}
$$

when $\nu=n+1 / 2=k a \cosh \gamma$ and $\gamma$ is positive and real.

Using the above approximations the coefficients $a_{n}$ and $b_{n}$ become

$$
\begin{aligned}
a_{n} & \sim\left[\frac{i \sinh \gamma_{1}+\left(\mu^{\prime} / N\right) \sinh \gamma \tanh \left(\psi_{2}-\psi_{1}\right)}{-i \sinh \gamma_{1}+\left(\mu^{\prime} / N\right) \sinh \gamma \tanh \left(\psi_{2}-\psi_{1}\right)}\right] \exp 2 \nu(\tanh \gamma-\gamma) \\
b_{n} & \sim\left[\frac{i \sinh \gamma_{1}+\left(N / \mu^{\prime}\right) \sinh \gamma \operatorname{coth}\left(\psi_{2}-\psi_{1}\right)}{-i \sinh \gamma_{1}+\left(N / \mu^{\prime}\right) \sinh \gamma \operatorname{coth}\left(\psi_{2}-\psi_{1}\right)}\right] \exp 2 \nu(\tanh \gamma-\gamma) .
\end{aligned}
$$

For $n$ sufficiently greater than $k a$, the exponents in the above expression are large and negative. Hence provided that the denominators of the expressions given by (25) and (26) do not become too small, the coefficients $a_{n}$ and $b_{n}$ for $n$ sufficiently greater than $k a$, are negligible. In consideration of the denominators it can be shown that since the real part of $\left(\psi_{2}-\psi_{1}\right)$ is positive and greater than the real part of $-i k_{1} \delta$, then the real part of coth $\left(\psi_{2}-\psi_{1}\right)$ is positive and with respect to magnitude, is greater than the imaginary part of coth $\left(\psi_{2}-\psi_{1}\right)$. In particular as $n$ approaches $\infty$, coth $\left(\psi_{2}-\psi_{1}\right)$ approaches unity. A similar analysis holds for tanh $\left(\psi_{2}-\psi_{1}\right)$. Hence if

$$
\left|\arg \left(N / \mu^{\prime}\right)\right| \leq \frac{\pi}{4}
$$


then

$$
\begin{aligned}
& \text { Real }\left\{\left(\mu^{\prime} / N\right) \tanh \left(\psi_{2}-\psi_{1}\right)\right\}>0 \\
& \text { Real }\left\{\left(N / \mu^{\prime}\right) \operatorname{coth}\left(\psi_{2}-\psi_{1}\right)\right\}>0 .
\end{aligned}
$$

In addition since the imaginary part of $\sinh \gamma_{1}$ is positive, then

$$
\text { Real }\left\{-i \sinh \gamma_{1}\right\}>0 \text {. }
$$

Hence it is easily seen that the real parts of the denominators of the right-hand sides of expressions (25) and (26) are greater than zero. Condition (27) can be relaxed to permit a greater range of permissible values for the argument of $N / \mu^{\prime}$, but only after considerable and extensive analysis.

Considering the corresponding problem for the coefficients of the form given by (21) and (22) one obtains for $n>M k a$

$$
\begin{aligned}
& a_{n} \sim\left(\frac{-1+i \eta \sinh \gamma}{1+i \eta \sinh \gamma}\right) \exp 2 \nu(\tanh \gamma-\gamma) \\
& b_{n} \sim\left(\frac{-i \eta-\sinh \gamma}{i \eta-\sinh \gamma}\right) \exp 2 \nu(\tanh \gamma-\gamma) .
\end{aligned}
$$

Provided that $\eta$ is bounded away from the imaginary axis, these behave like exponentials with large negative exponents.

Thus the Mie series for the coated sphere may be approximated by the Mie series for the boundary condition on the outer surface given by eq (6) with

$$
\eta=-\frac{i \mu^{\prime}}{N} \tan \left(k_{1} \delta\right)
$$

\section{High-Frequency Backscattered Field}

For the evaluation of the bistatic and forward-scattering far fields in the high-frequency range, it is best to express the scattered field in terms of the Debye potentials. However, for the case of backscattering one can work directly with the expression for the electric intensity.

From eq (1) the backscattered field is given by

$$
E^{s}=E_{0} \frac{e^{i k r}}{k r}(-i) \sum_{n=1}^{\infty}(-1)^{n}(n+1 / 2)\left(a_{n}-b_{n}\right) .
$$

Simplification is achieved for the sphere with a thin coating of large refractive index if the approximations to the coefficients $a_{n}$ and $b_{n}$ given by eqs (21) and (22) are used.

Combining the two coefficients and using the Wronskian relations for the Bessel functions, one obtains

$$
a_{n}-b_{n}=\frac{1-\eta^{2}}{\eta}\left[L_{n+1 / 2}(\eta) L_{n+1 / 2}(1 / \eta)\right]^{-1}
$$

where

$$
L_{n+1 / 2}(\eta)=i \eta \zeta_{n}^{(1)}(k a)+\zeta_{n}^{\prime(1)}(k a) .
$$

Treating the summation over $n$ as a residue series, the summation is replaced by a contour integral $C$ taken in the clockwise direction around the poles at $\nu=1 / 2,3 / 2, \ldots$, giving

$$
E^{s}=E_{0} \frac{e^{i k r}}{k r}\left\{\frac{i}{2}\left(a_{0}-b_{0}\right)-\frac{\left(1-\eta^{2}\right)}{2 \eta} \int_{c} \frac{\nu}{\cos \nu \pi L_{\nu}(\eta) L_{\nu}(1 / \eta)} d \nu\right\}
$$

The contour integral can be deformed using the Watson transform technique to give a line 
integral extending from the fourth quadrant through the origin to the second quadrant in the $\nu$ plane, plus a residue summation produced by the poles of $L_{\nu}(\eta)$ and $L_{\nu}(1 / \eta)$ in the first quadrant. The details of this well-known technique will not be discussed here.

An asymptotic evaluation of the line integral can be performed in the same manner as the technique of Scott [1949]. The combination of the asymptotic expression for the line integral and the term involving $\left(a_{0}-b_{0}\right)$ in the expression (32) gives the geometric optics contribution to the backscattered field, namely

$$
E_{\text {g. o. }}^{s} \sim\left(\frac{1-\eta}{1+\eta}\right)\left(\frac{-a}{2 r}\right)\left[1-\frac{i}{2 k a}+\ldots\right] e^{i k r-2 i k a} E_{0} .
$$

The residue series corresponding to the "creeping waves" or diffracted field contribution to the backscattered field is

$$
E_{d}^{s} \sim-\frac{e^{i k r}}{k r} E_{0}\left(\frac{1-\eta^{2}}{\eta}\right) i \pi\left\{\sum_{l=1}^{\infty} \frac{\nu_{l}}{\cos \nu_{l} \pi}\left[\frac{\partial L_{\nu}(\eta)}{\partial \nu} L_{\nu}(1 / \eta)\right]_{\nu=\nu_{l}}^{-1}+\sum_{s=1}^{\infty} \frac{\nu_{s}}{\cos \nu_{s} \pi}\left[L_{\nu}(\eta) \frac{\partial}{\partial \nu} L_{\nu}(1 / \eta)\right]_{\nu=\nu_{s}}^{-1}\right\}
$$

where $\nu_{l}$ and $\nu_{s}$ are the zeros of $L_{\nu}(\eta)$ and $L_{\nu}(1 / \eta)$ respectively. Since the dominant contribution arises from the zeros which are close to $k a$, the following asymptotic approximation is made,

where

$$
\begin{aligned}
& \zeta_{\nu-1 / 2}^{(1)}(k a) \sim-i m^{1 / 2} w_{1}(t) \\
& \zeta_{\nu-1 / 2}^{(1)}(k a) \sim i m^{-1 / 2} w_{1}^{\prime}(t)
\end{aligned}
$$

$$
m=\left(\frac{k a}{2}\right)^{1 / 3} \text { and } \nu=k a+m t .
$$

Here the notation of Fock [1946] is used for the Airy integral $w_{1}(t)$. It is related to the notation of Miller [1946], which is more useful for numerical results, by the following expression,

$$
w_{1}(t)=\sqrt{\pi}\left[B_{i}(t)+i A_{i}(t)\right] .
$$

For further reference on the subject, one should consult Logan [1959]. Excluding the case where $\eta=0$, which corresponds to the perfect conducting sphere, whose results are well-known, the residue series can be approximated by

$$
-\frac{e^{i k r}}{k r} E_{0} \frac{\pi}{m}\left\{\sum_{l=1}^{\infty} \frac{\nu_{l}}{\cos \nu_{l} \pi}\left[\left(\eta^{2}+\frac{t_{l}}{m^{2}}\right)\left(w_{1}\left(t_{l}\right)\right)^{2}\right]^{-1}-\sum_{s=1}^{\infty} \frac{\nu_{s}}{\cos \nu_{s} \pi}\left[\left(\frac{1}{\eta^{2}}+\frac{t_{s}}{m^{2}}\right)\left(w_{1}\left(t_{s}\right)\right)^{2}\right]^{-1}\right\}
$$

where $t_{l}$ and $t_{s}$ are respectively the roots of

and

$$
\frac{w_{1}^{\prime}(t)}{w_{1}(t)}=i \eta m
$$

$$
\frac{w_{1}^{\prime}(t)}{w_{1}(t)}=i m / \eta
$$

The values of $w_{1}^{\prime}(t) / w_{1}(t)$ have been plotted by Logan and Yee for values of $t$ in the first quadrant. From this the first few roots of the eqs (38) and (39) can be obtained.

The total backscattered field is obtained by combining expressions (33) and (37).

The reduction of the original problem (that of scattering by a perfectly conducting sphere with a thin coating of material of large index of refraction) to the equivalent problem (where the boundary condition is expressed in terms of an impedance boundary condition) greatly reduces the effort in obtaining the small wavelength asymptotic expression for the far scattered field. A similar technique should be applicable to other coated bodies with large radii of curvature. 


\section{References}

Aden, A. L., and M. Kerker, Scattering of electromagnetic waves from two concentric spheres, J. Appl. Phys. 2\%, No. 10, 1242 (1951).

Fock, V., The field of a plane wave near the surface of a conducting body, Journal of Physics USSR 10, No. 5, 399 (1946).

Hiatt, R. E., T. B. A. Senior, and V. H. Weston, A study of surface roughness and its effect on the backscattering cross section of spheres, Proc. I. R. E. 48, No. 12, 2008 (1960).

Logan, N. A., General research in diffraction theory, Vol. 1, Lockheed Tech. Rep. LMSD-288087 (1959).

Logan, N. A., and P. Blacksmith (ed.), Diffraction, refraction, and reflection of radio waves, Thirteen papers by V. A. Fock, Astia Doc. No. AD117276 (1957).

Miller, J. C. P., The Airy integral, giving tables of solutions of the differential equation $y^{\prime \prime}=x y$ (Univ. Press, Cambridge, 1946).

Scharfman, H., Scattering from dielectric coated spheres in the region of first resonance, J. Appl. Phys. 25, No. 11, 1352 (1954).

Scott, J. M. C., An asymptotic series for the radar scattering cross section of a spherical target, (British) A. E. C. Research Est. Rept. T/M 30 (1949).

Wait, J. R., Radiation from a vertical antenna over a curved stratified ground, J. Research NBS 56, No. 4, 237 (1956).

Watson, G. N., The diffraction of electric waves by the earth, Proc. Roy. Soc. London 95, 83 (1918).

Watson, G. N., A treatise on the theory of Bessel functions (Univ. Press, Cambridge, 1958).

Yee, K. S., and N. A. Logan, On a solution of the Riccati differential equation satisfied by the logarithmic derivative of the Airy function (to be published).

(Paper 66D5-222) 